\title{
The Radiographic Findings in Travelers with Chest Trauma Referred to a Tertiary Hospital in South Khorasan, Iran
}

\author{
Mahyar Mohammadi-Fard ${ }^{1}$, Mohammad-Mahdi Khalesi², Amin Saburi ${ }^{3}$, ${ }^{2}$, Kourosh Javdan ${ }^{1}$, Ghodratollah Naseh ${ }^{1}$ \\ ${ }^{1}$ Radiology Department, Faculty of Medicine, Birjand University of Medical Sciences, Birjand, Iran \\ ${ }^{2}$ Student Research Committee, Faculty of Medicine, Birjand University of Medical Sciences, Birjand, Iran \\ ${ }^{3}$ Health Research Center, Baqiyatallah University of Medical Sciences, Tehran, Iran
}

*Corresponding Author: Amin Saburi, MD, Health Research Center, Baqiyatallah University of Medical Sciences, Tehran, Iran Email: aminsaburi@yahoo.com

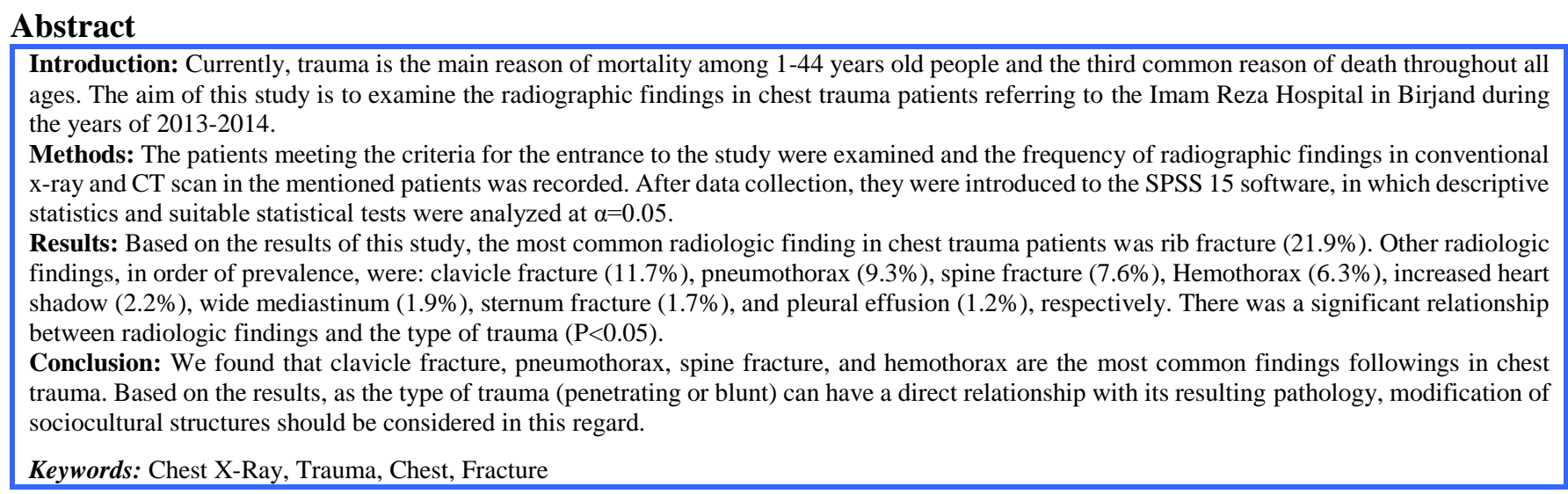

Article History: Received: 31 Mar. 2015; Accepted: 2 May. 2015; Online Published: 24 Aug. 2015

Cite this article as: Mohammadi-Fard M, Khalesi M, Saburi A, Javdan K, Naseh G. The radiographic findings in travelers with chest trauma referred to a tertiary hospital in south Khorasan, Iran. Int J Travel Med Glob Health. 2015;3(3):111-4.

\section{Introduction}

Currently, trauma including car accidents and traffic injuries is the main reason of mortality among 1-44 year old people and is the third common reason of death throughout all ages. The main reason of chest injury is accidents caused by vehicles $[1,2]$. One could say that trauma brings the greatest socioeconomic impact on all of the life stages of a person, and thus nowadays victims of trauma are studied more than other diseases [3]. In two thirds of vehicle accidents with damages to the chest, rib fracture is mostly observed [4-5]. In addition, in various studies it has been reported that $15-20 \%$ of victims have hidden damage of diaphragm through lower penetrating trauma of the chest [6]. A statistical analysis in the US has indicated that damages to the chest accounts for $25 \%$ of all mortality cases caused by trauma, out of which around $50 \%$ have occurred due to fatal accidents [7, 8]. Recently, thoracoscopy has been introduced as a measure with slight intrusion and high accuracy for investigation, diagnosis, and treatment of diaphragm traumas $[9,10]$. Chest radiography is the preliminary test for all people suffering from non-penetrating trauma of chest $[1,11$, 12]. Nowadays, with the advances emerged in the transportation of injured patients to hospitals and in presentation of medical services to these patients, it seems that the number of these injured individuals who reach hospitals alive is constantly increasing. Improvement of emergency services and rapid transfer of the injured to hospitals have increased the number of recovered patients $[13,14]$. Almost in all references, the significance of early diagnosis and treatment has been emphasized in order to diminish the complications and mortalities [13, 14]. The emergency physician should also deal with injured patients immediately by knowing the pathophysiological principles of trauma to the chest. It is possible to reduce the rate of mortality and morbidity by proper diagnosis of the type of damage to the chest. In both types of penetrating and nonpenetrating traumas, one should consider the damages accompanying chest injuries as well [15]. The objective of this study is the examination of radiographic findings in chest trauma patients referring to Imam Reza hospital during 2013-2014. This was done in order to find a suitable framework for taking timely and proper therapeutic measures when dealing with these patients.

\section{Methods}

This cross-sectional study was conducted on 410 patients with trauma referring to Imam Reza Hospital in Birjand in 2013-2014. Their chest was injured and accordingly their physician requested chest radiography.

The criteria of entrance to this study included: chest trauma, request of chest radiography by the physician.

The criteria of exclusion from the study included: death of the patient before taking preliminary measures, leaving emergency with personal consent before conductance of diagnostic measures.

The selection of patients was done based on nonprobabilistic sampling method. The patients participating in this study were suffering from chest trauma due to multiple trauma, accident, collapsing from height, accident during work, quarrel, etc. who referred to Imam Reza Hospital. At first, a thorough description was taken from the patient themselves or if unconscious, from their companions or 
EMS. Next, if necessary, the measures of resuscitation and the stabilization of the patient were taken. Complimentary examinations were further carried out. These examinations included chest radiography, ECG, EEG, CT Scan, Blood and urine tests, etc. with the help of these tests, the diagnosis was made based on which proper therapeutic measures were taken.

For every referring patient, a questionnaire was filled in which the gender, age, the way damage was incurred, the background of any special disease, similar accidents, etc. were included. After collection of these information, findings, and the results obtained from the history, examinations, tests, and paraclinical investigations, the frequency of the radiographic findings in the mentioned patients was studied during the research.

\section{Results}

In this study, $84.6 \%$ of the patients were male and $15.4 \%$ were female.

The age of the patients was divided into four groups: 0-10 years, 10-30 years, 30-50 years, and 50 years above.

The highest frequency percentage belonged to the 10-30 year-old age group (48\%), while the lowest was related to the above-50-years age group $(13.2 \%)$.

The patients participating in this study were examined in terms of the type of trauma (penetrating or blunt) and were then compared in terms of gender.

The frequency percentage of penetrating trauma was $23.2 \%$ and the blunt type was $76.8 \%$.

The comparison of these two groups using the Chi-Square statistical test indicated a significant relationship between the type of trauma and gender $(\mathrm{p}<0.05)$. Both penetrating and blunt trauma were far more prevalent in males than in females.

A background of trauma to the chest, cardiovascular diseases, and respiratory diseases was investigated in the studied patients. $4.6 \%$ mentioned a background of trauma to the chest, $6.8 \%$ had a cardiovascular disease in their background, and 9\% had a background of respiratory diseases.

The type of requested graphy (AP or PA) was also investigated in this study. The posterior-anteriorgraphy was requested with a higher prevalence $(76.1 \%)$.

Table 1. Radiologic findings among injured travelers

\begin{tabular}{lcc}
\hline \multicolumn{1}{c}{ Findings } & Frequency & Percentage \\
\hline Rib fracture & 90 & 21.9 \\
Clavicle fracture & 48 & 11.7 \\
Spinal fracture & 31 & 7.6 \\
Sternal fracture & 7 & 1.7 \\
C/T Ration increase & 9 & 2.2 \\
Wide Mediastinum & 8 & 1.9 \\
Pleural effusion & 5 & 1.2 \\
Pneumothorax & 38 & 9.3 \\
Hemothorax & 26 & 6.3 \\
\hline
\end{tabular}

Table 2. Chest CT findings among traumatic travelers

\begin{tabular}{lcc}
\hline \multicolumn{1}{c}{ Findings } & Frequency & Percentage \\
\hline Soft tissue injuries & 15 & 3.6 \\
Pulmonary contusion & 13 & 3.2 \\
Main vessels injuries & 6 & 1.5 \\
Aortic dissection & 0 & 0 \\
Hemothorax & 14 & 3.4 \\
Subcutaneous & 8 & 1.9 \\
emphysema & 11 & 2.7 \\
Pneumothorax & & \\
\hline
\end{tabular}

The radiologic findings were investigated in the studied patients with the results provided in Table 1.

The most common radiologic finding in chest trauma patients was rib fracture $(21.9 \%)$.

In the radiographic cliché of $58 \%$ of patients (238 patients), no radiologic finding was observed.

The frequency distribution of the radiologic findings in the studied patients was investigated in terms of gender. No significant relationship was seen between various radiologic findings and the gender ( $\mathrm{p}>0.05)$.

The frequency distribution of the radiologic findings in the studied patients was investigated in terms of age. No significant relationship was seen between various radiologic findings and the age group ( $\mathrm{p}>0.05)$.

The frequency distribution of the radiologic findings in the studied patients was investigated in terms of the type of trauma. There was a significant relationship between various radiologic findings and the type of trauma $(\mathrm{p}<0.05)$.

In 37 patients $(9 \%)$, CT-Scan was requested with its findings provided in Table 2.

The most frequent finding in CT scan was damage to soft tissue $(3.6 \%)$.

\section{Discussion}

This study was conducted with the aim of investigating the radiologic findings in chest trauma patients visiting the Imam Reza educational Hospital in Birjand in 2013-2014. This was done in order to find a suitable framework for taking timely and proper therapeutic measures when dealing with these patients.

Based on the findings of this study, $84.6 \%$ of patients who had chest trauma were male and $15.4 \%$ were female. This finding is congruent with similar studies where in numerous investigations this frequency has been reported to be $75-89 \%$ in males and $12-25 \%$ in females [16-18].

In this study, the highest and lowest frequency belonged to the patients in the age group of 10-30 (48\%) and above 50 $(13.2 \%)$ years of age, respectively. This finding was in accordance with the studies by Beyzani et al, done in Fasa town together with Ahmadi Amoli et al. which had been conducted in three therapeutic centers in Tehran to examine chest trauma [16, 19].

Based on the data of this investigation, the frequency percentage of penetrating and blunt traumas were 23.2 and $76.8 \%$, respectively.

Similarly, both types of penetrating and blunt traumas were far more frequent in males than females. In a research by Dr. Zohreh Haratian et al, the prevalence of penetrating and blunt traumas were $21.9 \%$ and $78.1 \%$, respectively congruent with this study [15].

Likewise, in the study by Bijani et al, the prevalence of penetrating and blunt traumas were 20 and $80 \%$, respectively, in accordance with the present study [19].

In a similar vein, in the study by Ahmadi Amoli, the prevalence of penetrating and blunt traumas was 24 and $76 \%$, respectively, very similar to the results of this study [16].

Based on the results of this study, 4.6, 6.8, and $9 \%$ of patients mentioned a background of trauma to chest, cardiovascular diseases, and respiratory diseases, respectively. In this regard, no other study addressing these indices were found. However, considering the prevalence rate of these diseases in the region and the mean age of the studied patients, these values can be considered acceptable. 
In this study, posterior-anterior graphy was requested with a higher frequency $(76.1 \%)$. Numerous studies confirmed this result including the studies conducted by Stuart et al. [23], Wiki et al. [17], Rasmusen et al. [18], and Wegner et al. [20]. Based on the results of this study, the most common radiologic finding in the patients suffering from chest trauma was chest fracture $(21.9 \%)$.

Other radiologic findings in the order of prevalence are as follows:

Clavicle fracture $(11.7 \%)$, pneumothorax $(9.3 \%)$, fracture of spine $(7.6 \%)$, hemothorax $(6.3 \%)$, increased heart shadow $(2.2 \%)$, wide mediastinum $(1.9 \%)$, sternum fracture $(1.7 \%)$, and pleural effusion $(1.2 \%)$. The order of prevalence of the above findings was in accordance with the study by Bijani et al. in the Fasa town [19].

The prevalence of some findings in other studies was significantly different possibly due to regional differences and the discrepancies between common traumas along with different statistical population $[15,21,22]$.

In this study, the participants had experienced no injuries. This can be due to two reasons: the studied profiles were chosen from outpatients and inpatients, where outpatients had no serious problems. Most incurred damages were due to driving accidents and accordingly the reason of visiting medical centers has been seeking reassurance about the health of an injured person in order to prevent incidences of further legal problems.

CT scan was performed on 37 patients (9\%). In CT, the most frequent injury was related to soft tissues (3.6\%) followed by hemothorax $(3.4 \%)$. In this investigation, the crushing of lungs (3.2\%), pneumothorax (2.7\%), and subcutaneous emphysema $(1.9 \%)$ were the next common injuries in the CT. In a similar study in Turkey, pneumothorax and hemothorax were reported to be 6 and $7 \%$ in patients, respectively.

\section{Conclusion}

According to the results obtained from this study, it can be concluded that fractureof chest, clavicle, pneumothorax, spine fracture, and hemothorax are the most prevalent pathologies following a trauma to the chest. Therefore, proper and timely diagnostic and therapeutic methods and especially approaches to preventing the incidence of these injuries are of significance. Furthermore, as the type of trauma can be directly related to pathologies resulting from that, reformation of sociocultural structures should be considered as well.

Similarly, proper planning for prevention from incidence of these damages should be considered in all age groups especially in children and the elderly. Because, based on the results of this study, damages caused by trauma were observed throughout all ages even in these two vulnerable groups with a relatively high prevalence.

Since most severely injured people lose their lives before arriving at medical centers, thus public education and giving instruction to special rescue teams and police with regard to the way traumatic patients should be dealt with can improve the quality and speed of therapeutic interventions. Similarly, this can be of great help in transferring the patients as quickly as possible to medical centers and construction of specialist centers of trauma.

One limitation of this study was the lack of investigation of the intensity of trauma due to the nature and type of the study. Moreover, determination of the number of hospitalization days and the need for an artificial respiration device as well as the determination of the place and reasons of requiring surgical interventions were the other limitations. It is suggested that these under-investigated issues be further studied in the future.

\section{Acknowledgments}

We highly appreciate the financial and spiritual support of the research and technology deputy of Birjand University of Medical Sciences.

\section{Authors' Contributions}

All authors had a role in designing the study, data interpretation and drafting the article. Mohammadi-Fard and Khalesi gathered the data. At the end, all the authors approved the final draft.

\section{Financial Disclosure}

This study was conducted under the financial support of the Faculty of Medicine, Baqiyatallah University of medical sciences, Tehran, Iran as a Medical Student thesis.

\section{Funding/Support}

This study was support by the Faculty of Medicine, Baqiyatallah University of medical sciences, Tehran, Iran.

\section{References}

1. Liman ST, Kuzucu A, Tastepe AI, Ulasan GN, Topcu S. Chest injury due to blunt trauma. Eur J Cardiothorac Surg. 2003;23(3):374-8

2. Rodriguez RM, Hendey GW, Marek G, dery RA, Bjoring A. A pilot study to drive clinical variables for selective chest radiography in blunt trauma patients. Ann Emerg Med. 2006;47(5):415-8

3. Kish G, Kozlof L, Joseph WK, Kevstian D. Indications for early thoracotomy in the management of chest trauma. Ann Thorac Surg. 1997;22:23-8

4. Kohn MA, Hammel JM, Bretz SW, Stangby A. Trauma team activation criteria as predictors of patient disposition from the emergency department. Acad Emerg Med. 2004;11(1):1-9

5. Newman RJ, Jones IS. A prospective study of 413 consecutive car occupants with chest injuries. J Trauma. 1984;24(2):129-35.

6. Hegarty M, Brown GL, Richardson JD. Traumatic diaphragm hernia. Sheilds T, Locicero J, Pom R. General Thoracic Surgery. 5thed. Philadelphia: Lippincott Williams \& Wilkins; 2004. p. 866-70.

7. Blair E, Topuzulu C, Dean RS. Major chest trauma. CurrProblSurg 1962;2:69-73

8. Krish MM, Sloan H. Blunt chest trauma: general principles of management .2nd edition, Boston, Little Broun 1997; p. 297-314

9. Jackson AM, Ferreira AA. Thoracoscopy as an aid to the diagnosis of diaphragmatic injury in penetrating wounds of the left lower chest: a preliminary report. Injury. 1976;7:213-7.

10. Smith RS, Fry WR, Tsoi EK, Morabito DJ, Koehler RH, Reinganum $\mathrm{SJ}$, et al. Preliminary report on videothoracoscopy in the evaluation and treatment of thoracic injury. Am J Surg. 1993;166:690-3.

11. Marnocha KE, Maglinte DD, Woods J, Peterson PC, Dolan PA, Nigh A, et al. Blunt chest trauma and suspected aortic rupture: reliability of chest radiograph findings. Ann Emerg Med. 1985;14(7):644-9

12. Kram HB, Appel PL, Wohlmuth DA, Shoemaker WC. Diagnosis of traumatic thoracic aortic rupture: a 10 -year retrospective analysis. Ann Thorac Surg. 1989;47(2):282-6

13. Baumgarthner F, Sheppard B, Virigilio C, Esrig B, Harrier D, Nelson RJ, et al. Tracheal and main bronchial disruptions after blunt chest trauma: Presentation and management. Ann Thracsurg. 1990;50:56974.

14. Cassada DC, Munyikwa MP, Moniz MP, Dieter RA, Schmann GF, Enderson BL. Acute injuries of the trachea and major bronchi: Importance of early diagnosis. 1990; p. 1563-67.

15. Haratian Z. Study of the prevalence of damages caused by chest trauma (penetrating or blunt) in the beast hospital of Air force 20012004. Med J Azad Univ. 2005;15(3):147-50. Persian

16. Ahmadi Amoli H, Zafarqandi M, Tavakoli H. Chest trauma: assessment of injury in 342 patients. J Tehran Univ Med Sci. 2007;66(11):831-4. Persian

17. Wicky S, Wintermark M, Schnyder P. Imaging of blunt chest trauma. 
Eur Radiol. 2000;10(10):1524-38.

18. Rasmussen OV, Brynitz S, Struve-Christensen E. Thoracic injuries. A review of 93 cases. Scand J Thorac Cardiovasc Surg. 1986;20(1):71-4.

19. Bijani M, Nikruz L, Naqizade M, Tavakol Z. study of people with chest trauma referred to Valiasr hospital in Fasa. J Fasa Univ Med Sci. 2013;3(3):285-9. Persian

20. Van Wagoner FH. Died in hospital; A three-year study of deaths following trauma. J Trauma. 1961;1:401-8.
21. Golestanha A, Jabarimoqadam Y. Assessment of radiologic findings of extremities in war injured patients. JAUMS. 2008;5(4):1419-22.

22. Shafinia R. Study of prevalence of radiologic findings in people with blunt chest trauma who refered to azzahra and kashani emergency sections in winter 2003. J Tehran Univ Med Sci. 1382;89(1):28. Persian

23. Stewart RM, Myers JG, Dent DL, Erms Gray GA, Villarreal R, et al. Seven hundred fifty three consecutive deaths in a trauma center: the argument for injury prevention. J Trauma. 2003;54(1):66-70. 